\title{
Author Correction: The softness of tumour-cell-derived microparticles regulates their drug-delivery efficiency
}

Qingle Liang, Nana Bie, Tuying Yong, Ke Tang, Xiaolong Shi, Zhaohan Wei, Haibo Jia, Xiaoqiong Zhang, Haiyan Zhao, Wei Huang, Lu Gan D, Bo Huang (D) and Xiangliang Yang (D)

Correction to Nature Biomedical Engineering https://doi.org/10.1038/s41551-019-0405-4, published online 20 May 2019.

In the version of this Article originally published, the Supplementary Information file contained two errors. In Supplementary Fig. 17d, the $\mathrm{IC}_{50}$ values of free DOX for the H22 cells were mistakenly identical to those for the B16-F10 cells. The correct values have now been provided and the associated $P$ values have also been corrected. Additionally, in Supplementary Fig. 23a, the day- 6 representative spheroid for the top row (peripheral region) was mistakenly a duplicate of the day- 5 representative spheroid for the bottom row (central region). The correct spheroid image has now been provided, and the updated Supplementary Information file is available online.

Published online: 8 February 2021

https://doi.org/10.1038/s41551-021-00694-0

(C) The Author(s), under exclusive licence to Springer Nature Limited 2021 